\title{
PULSATIONS OF WOLF-RAYET STARS: OBSERVATIONAL RESULTS FOR FIVE WR STARS
}

\author{
P. BRATSCHI \\ Geneva Observatory, CH-1290 Sauverny, Switzerland
}

\begin{abstract}
Since the recent discovery of fast pulsations of a WR star (Blecha et al. 1992), we observed five WR stars intensively and we report here the detection of fast photometric variations for three of them. These variations have a quasi periodic features with a timescale compatible with theoretical predictions (Maeder 1985, Glatzel et al. 1993). For all observed WR stars the period can vary significantly and even disappear, showing the erratic nature of this phenomenon.
\end{abstract}

Key words: stars: Wolf-Rayet - stars: mass loss - photometry

\section{Introduction}

Models of WR stars indicate that the fundamental radial mode could be excited due to nuclear-burning instabilities with a period of few minutes (Maeder 1985; Cox and Cahn 1988). These instabilities would be the source of the mechanical energy powering the strong stellar wind, whose acceleration mechanism is still not understood. Encouraged by the discovery of the first pulsating WR star, we started an intensive programme of UBV Geneva photometry of WR stars, although the pulsation of WR40 have not been confirmed by other observers (Marchenko et al. 1994, Schneider et al. 1993, Martinez et al. 1993). Our CCD camera does not allow the simultaneous observation of a comparison star and the WR star. Therefore to check the stability of instrumentation and atmospheric conditions, we observed a stable standard star during approximatively 20 minutes before and after observing the WR stars (Blecha et al. 1992, Fig.1).

\section{Results and discussion}

HD50896 - WR6 (WN5). WR6 is undoubtedly a fast variable star, but without well defined periodicity. It shows features like pulsations (Fig.1.), sudden variations or stability, closer to an erratic than regular behaviour.

HD96458 - WR40 (WN8). We could re-observe this star only in January 94 and we did not see any variation, as Schneider et al. (1994), Marchenko et al. (1994) and Martinez et al. (1994).

HD151932 - WR78 (WN7). WR78 showed quasi periodic variations (Fig.1) HD165763 - WR111 (WC5). This star showed clear periodic variations during 3 nights. The period is about 20 minutes and with a big enough amplitude to be undubitable (Fig.1)

HD76536 - WR14 (WC6). This WC star, did not show any variation. 

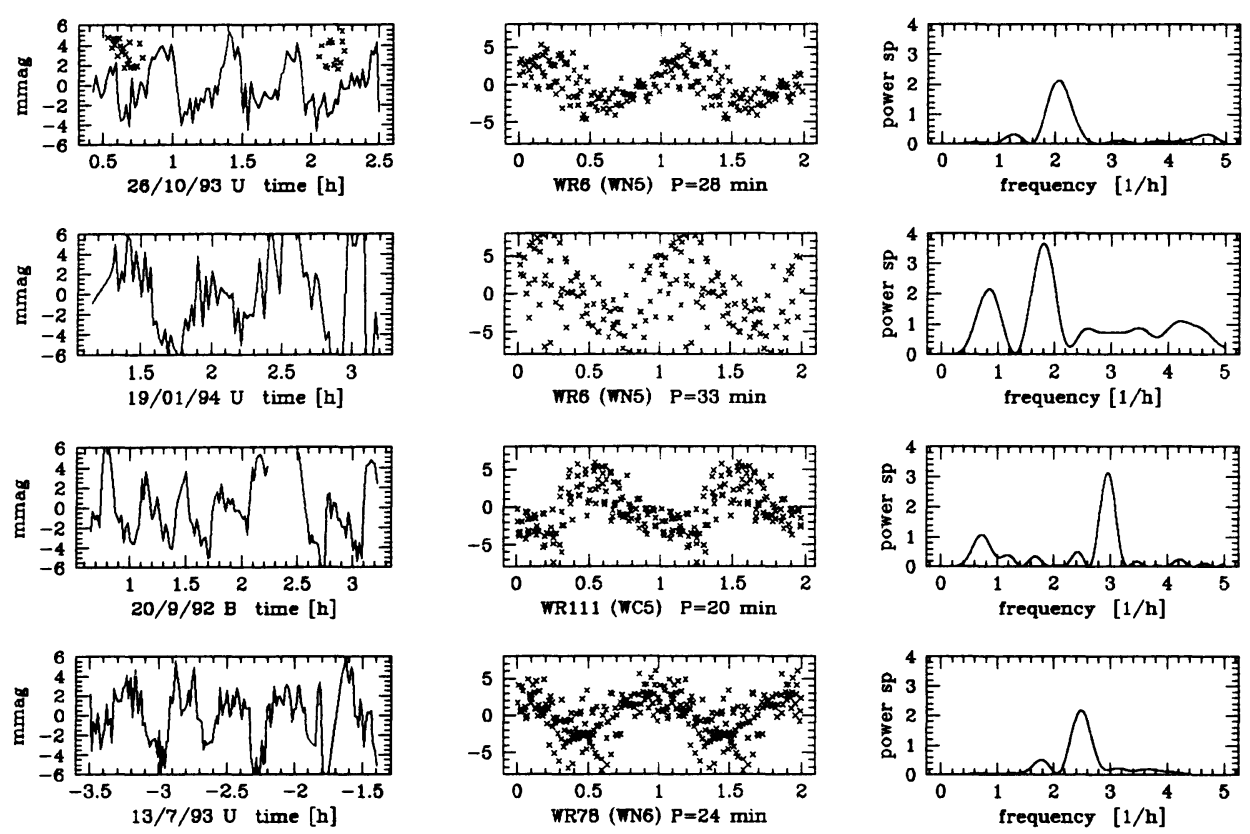

Fig. 1. Light-curves for three WR stars (a standard star is showed above)

\section{Conclusion}

Analyses of linear models show that pure $\mathrm{He}$ and $\mathrm{He}+\mathrm{C}+\mathrm{O}$ stars with a mass above the critical mass are the seat of vibrational instabilities (Maeder 1985; Cox and Cahn 1988). These pulsations have periods of about 30 minutes and may be the source of the mechanical energy responsible for the stellar wind acceleration. The observations show that WR stars are varying rapidly but mostly with an erratic behaviour. Presents models and instabilities analysis describe the steady state of the star pulsation (Schaller 1992) and can not describe or predict the transient phases. Furthermore these models do not take into account the interactions between the star and its wind. Our observations rather suggest series of instabilities or pulsations interrupted by quieter phases.

\section{References}

Blecha, A., Schaller, G., Maeder, A. 1992, Nature 360, 320

Cox, A., Cahn, J. 1988, ApJ 326, 804

Maeder, A., 1985, $A \mathscr{E} A$ 147, 300

Martinez, P., Kurtz, D., Ashley, R., Tripe, P. 1994, Nature 367, 601

Marchenko, S. et al. 1994, in press

Glatzel, W., Kiriakidis, M., Fricke, K.J. 1993, MNRAS 262, P7

Schaller, G. 1992, PhD. thesis, Université de Genève

Schneider, H., Kiriakidis, M., Weiss, W., Glatzel, W., Fricke, K.J. 1994, in press 